\title{
ANY FLAT BUNDLE ON A PUNCTURED DISC HAS AN OPER STRUCTURE
}

\author{
EdWARd Frenkel AND Xinwen Zhu
}

\begin{abstract}
We prove that any flat $G$-bundle, where $G$ is a complex connected reductive algebraic group, on the punctured disc admits the structure of an oper. This result is important in the local geometric Langlands correspondence proposed in [7]. Our proof uses certain deformations of the affine Springer fibers which could be of independent interest. As a byproduct, we construct representations of affine Weyl groups on the homology of these deformations generalizing representations constructed by Lusztig.
\end{abstract}

\section{Introduction}

Let $G$ be a connected reductive algebraic group over $\mathbb{C}, \mathfrak{g}=\operatorname{Lie}(G)$. Let $F=\mathbb{C}((t))$ and $\mathcal{O}=\mathbb{C}[[t]]$. In this note we prove that every flat $G$-bundle on the formal punctured disc $D^{\times}=\operatorname{Spec} F$ has an oper structure. This proves Conjecture 10.1.1 of [4] (see also [5], Conjecture 1).

By definition, a flat $G$-bundle (equivalently, de Rham $G$-local system) on $D^{\times}$is a principal $G$-bundle on $D^{\times}$with a connection, which is automatically flat. In concrete terms, the set of isomorphism classes of flat $G$-bundles is the quotient

$$
\operatorname{Loc}_{G}\left(D^{\times}\right)=\mathfrak{g}(F) / G(F),
$$

where $G(F)$ acts on its Lie algebra $\mathfrak{g}(F)$ by gauge transformations as follows:

$$
\operatorname{Ga}_{g}(A)=\operatorname{Ad}_{g}(A)-\left(\partial_{t} g\right) g^{-1}, \quad \text { for } A \in \mathfrak{g}(F), g \in G(F) .
$$

The meaning of the expression

$$
d \log (g):=\left(\partial_{t} g\right) g^{-1}
$$

as an element in $\mathfrak{g}(F)$ is spelled out, e.g., in [4] §1.2.4.

Let $B \subset G$ be a Borel subgroup. We recall [2] that a $G$-oper is a flat $G$-bundle with a reduction to $B$ satisfying certain conditions. Let us describe the set of isomorphism classes of $G$-opers on $D^{\times}$in concrete terms. Choose a maximal torus $T \subset B$ and let $\mathfrak{t} \subset \mathfrak{b}$ be the corresponding inclusion of Lie algebras. Let $I_{\mathrm{f}}$ be the set of vertices in the finite Dynkin diagram corresponding to $G$. Let $\alpha_{i} \in \mathfrak{t}^{*}, i \in I_{\mathrm{f}}$ be the set of simple roots and $X_{-\alpha_{i}} \in \mathfrak{g}_{-\alpha_{i}}$ be a non-zero root vector corresponding to $-\alpha_{i}$. (Here, for a $\operatorname{root} \beta \in \mathfrak{t}^{*}$, we write $\mathfrak{g}_{\beta}$ for the corresponding root subspace of $\mathfrak{g}$.) Then the space of $G$-opers on $D^{\times}$is the quotient

$$
\mathrm{Op}_{G}\left(D^{\times}\right)=\left\{\sum_{i \in I_{\mathrm{f}}} \psi_{i} X_{-\alpha_{i}}+v \mid \psi_{i} \in F^{\times}, v \in \mathfrak{b}(F)\right\} / B(F),
$$

Received by the editors December 9, 2008. Revision received December 9, 2009.

Supported by DARPA and AFOSR through the grant FA9550-07-1-0543. 
where the action of $B(F)$ is given by (2). Note that if $G$ is semisimple of adjoint type, then $T(F)$ acts simply transitively on the space of the $\psi_{i}, i \in I_{\mathrm{f}}$. Hence the quotient (4) is isomorphic to

$$
\mathrm{Op}_{G}\left(D^{\times}\right)=\left\{\sum_{i \in I_{\mathrm{f}}} X_{-\alpha_{i}}+v \mid v \in \mathfrak{b}(F)\right\} / N(F),
$$

where $N=[B, B]$ is the unipotent radical of $B$.

There is an obvious forgetful map

$$
\mathrm{Op}_{G}\left(D^{\times}\right) \rightarrow \operatorname{Loc}_{G}\left(D^{\times}\right),
$$

taking the $B(F)$-gauge equivalent classes to $G(F)$-gauge equivalent classes.

The main result of this note is

Theorem 1. The map (6) is surjective.

This statement is important in the local geometric Langlands correspondence developed by D. Gaitsgory and the first author [7] (see [4] for an exposition). According to [7], to each flat ${ }^{L} G$-bundle $\sigma$ on $D^{\times}$one should be able to assign a category $\mathcal{C}_{\sigma}$ equipped with an action of the formal loop group $G(F)$ (here ${ }^{L} G$ is the Langlands dual group of $G$, which in this paragraph is assumed to be a simply-connected semisimple complex algebraic group, so that ${ }^{L} G$ is of adjoint type). These categories should satisfy some universality property. In [7] a candidate for $\mathcal{C}_{\sigma}$ was proposed. Namely, let $\chi$ be a pre-image of $\sigma$ in $\mathrm{Op}_{L_{G}}\left(D^{\times}\right)$under the map (6), with $G$ replaced by ${ }^{L} G$ (provided that it exists). Then $\mathcal{C}_{\sigma}$ should be equivalent to the category of modules over the affine Kac-Moody algebra $\widehat{\mathfrak{g}}$ of critical level with central character determined by $\chi$. This category is equipped with a natural action of $G(F)$. However, for this prescription to work for all $\sigma$ it is necessary for the map (6) to be surjective.

Remark 1. A flat $G L_{n}$-bundle on $D^{\times}$is the same as a rank $n$ vector bundle $\mathcal{F}$ on $D^{\times}$with a connection $\nabla .(\mathcal{F}, \nabla)$ has an oper structure if and only if there exists $\phi \in \Gamma\left(D^{\times}, \mathcal{F}\right)$ such that $\phi, \nabla \phi, \ldots, \nabla^{n-1} \phi$ generate $\mathcal{F}$. Such $\phi$ is called a cyclic vector of $(\mathcal{F}, \nabla)$. Therefore, the statement of Theorem 1 for $G=G L_{n}$ means that any flat rank $n$ vector bundle on $D^{\times}$has a cyclic vector. This statement is proved in [3], pp. $42-43$.

Remark 2. Let us recall Kostant's theorem [13]. Set $f=\sum_{i \in I_{\mathrm{f}}} X_{-\alpha_{i}}$. Kostant proved that every regular orbit of $\mathfrak{g}$ intersects with $f+\mathfrak{b}$. In other words, the map

$$
\{f+v \mid v \in \mathfrak{b}\} / N \rightarrow \mathfrak{g}^{\mathrm{reg}} / G
$$

is surjective (in fact, an isomorphism), where $\mathfrak{g}^{\text {reg }} / G$ denotes the GIT quotient. Therefore, Theorem 1 may be viewed as an analogue of Kostant's theorem for connections on the punctured disc (compare with formula (5)). An important difference is that a connection can be brought into an oper form without any regularity assumption.

Remark 3. The statement analogous to Theorem 1 for a smooth projective curve $X$ of genus greater than zero is known to be false. For instance, if $G$ is of adjoint type, there is a unique (up to an isomorphism) $G$-bundle on $X$ that can carry an oper structure (see [2] §3.5). However, it is expected that any flat $G$-bundle on $X$ has an oper structure with regular singularities at finitely many points. 


\section{Proof of the main theorem in the case when $A_{r}$ is regular nilpotent}

We begin our proof of Theorem 1. Let $A \in \mathfrak{g}(F)$. By taking $r$ small enough, we can always assume that $A$ may be written as

$$
A=A_{r} t^{r}+A_{r+1} t^{r+1}+\cdots, \quad r<-1, \quad A_{r} \text { is nilpotent. }
$$

Here $A_{r}$ can be zero. First, we have

Lemma 2. If $A_{r}$ is regular nilpotent, then there exists some $g \in G(\mathcal{O})$ such that $\operatorname{Ga}_{g}(A)$ is an oper.

Proof. Without loss of generality, we can, and will, assume that $A_{r}=f=\sum_{i \in I_{\mathrm{f}}} X_{-\alpha_{i}}$. Let $e \in \mathfrak{b}$ be the unique element such that $\{e, 2 \check{\rho}, f\}$ is a principal $\mathfrak{s l}_{2}$-triple. Let $\mathfrak{g}^{e}$ be the centralizer of $e$ in $\mathfrak{g}$. Let $G^{(1)}(\mathcal{O})$ be the first congruence subgroup of $G(\mathcal{O})$, i.e. the kernel of the evaluation map $G(\mathcal{O}) \rightarrow G$. We prove that there is some $g \in G^{(1)}(\mathcal{O})$ such that $\mathrm{Ga}_{g}(A) \in f t^{r}+\mathfrak{g}^{e}(F)$, which is in the oper form.

According to representation theory of $\mathfrak{s l}_{2}$, we have $\mathfrak{g}=\mathfrak{g}^{e}+\operatorname{ad} f(\mathfrak{g})$. Therefore, there exists $X_{1} \in \mathfrak{g}$ such that $A_{r+1}+\left[X_{1}, f\right] \in \mathfrak{g}^{e}$. Let $g_{1}=\exp \left(t X_{1}\right)$. Since $r<-1$,

$$
\mathrm{Ga}_{g_{1}}(A)=f t^{r}+\left(A_{r+1}+\left[X_{1}, f\right]\right) t^{r+1}+\widetilde{A}_{r+2} t^{r+2}+\cdots .
$$

Next, there exists some $X_{2} \in \mathfrak{g}$ such that $\widetilde{A}_{r+2}+\left[X_{2}, f\right] \in \mathfrak{g}^{e}$. Let $g_{2}=\exp \left(t^{2} X_{2}\right)$. Again, since $r<-1$,

$\operatorname{Ga}_{g_{2}}\left(\operatorname{Ga}_{g_{1}}(A)\right)=f t^{r}+\left(A_{r+1}+\left[X_{1}, f\right]\right) t^{r+1}+\left(\widetilde{A}_{r+2}+\left[X_{2}, f\right]\right) t^{r+2}+\widetilde{A}_{r+3} t^{r+3}+\cdots$

By induction, we can find $g_{1}, \ldots, g_{k-1}$ such that the coefficients of $t^{r+1}, \ldots, t^{r+k-1}$ of $\mathrm{Ga}_{g_{k-1}} \cdots \mathrm{Ga}_{g_{1}}(A)$ are in $\mathfrak{g}^{e}$. Let $\widetilde{A}_{r+k}$ be the coefficient of $t^{r+k}$ in $\mathrm{Ga}_{g_{k-1}} \cdots \mathrm{Ga}_{g_{1}}(A)$. Let $X_{k} \in \mathfrak{g}$ such that $\widetilde{A}_{r+k}+\left[X_{k}, f\right] \in \mathfrak{g}^{e}$ and let $g_{k}=\exp \left(t^{k} X_{k}\right)$. Then the coefficient of $t^{r+k}$ in $\mathrm{Ga}_{g_{k}} \ldots \mathrm{Ga}_{g_{1}}(A)$ belongs to $\mathfrak{g}^{e}$, while the coefficients of $t^{r}, \ldots, t^{r+k-1}$ remain unchanged. Let $g=\cdots g_{k} \cdots g_{2} g_{1}$. This is a well-defined element in $G^{(1)}(\mathcal{O})$ which satisfies the requirement of the lemma.

Remark 4. Let $A_{r}$ be an arbitrary regular element of $\mathfrak{g}$. By Kostant's theorem (see Remark 2), we can assume, without loss of generality, that $A_{r}=f+v, v \in \mathfrak{b}$. By a slight modification of the above argument, we can then also prove that there exists $g \in G^{(1)}(\mathcal{O})$ such that $\operatorname{Ga}_{g}(A)$ is an oper. Thus, we obtain a simple proof of the statement of Theorem 1 in the case when the leading term $A_{r}$ is regular. The real challenge is to prove that it holds even without this assumption.

By the previous lemma, in order to prove Theorem 1 it suffices to prove that there exists $g \in G(F)$ such that $B=\operatorname{Ga}_{g}(A)=B_{r} t^{r}+B_{r+1} t^{r+1}+\cdots$, with $B_{r}$ regular nilpotent. Recall that we are under the assumption $r<-1$. The rest of this paper is devoted to proving this fact.

\section{Deformed affine Springer fibers}

If $A=A_{r} t^{r}+A_{r+1} t^{r+1}+\cdots$ with $A_{r} \neq 0$, we call $r$ the order of $A$, and sometimes denote it by $\operatorname{ord}(A)$. Let

$$
M_{A}=\left\{g \in G(F) \mid \operatorname{ord}\left(\mathrm{Ga}_{g^{-1}}(A)\right) \geq \operatorname{ord}(A)=r\right\} .
$$


This is a subset of elements $g$ of $G(F)$ which is the set of solutions of certain algebraic equations on the coefficients of $g$. Hence it is clear that it is the set of points of an ind-subscheme of $G(F)$. It is clearly invariant under the right multiplication by elements of the subgroup $G(\mathcal{O})$. Therefore the quotient

$$
Y_{A}:=M_{A} / G(\mathcal{O})
$$

is a well-defined closed ind-subscheme of the affine Grassmannian $\operatorname{Gr}=G(F) / G(\mathcal{O})$. We call it the deformed affine Springer fiber associated to $A$.

Let us explain this terminology. Set $\widetilde{A}=t^{-r} A \in \mathfrak{g}(\mathcal{O})$. For $\lambda \in \mathbb{C}$, let

$$
Y_{\widetilde{A}, \lambda}=\left\{g \in G(F), \operatorname{Ad}_{g^{-1}}(\widetilde{A})-\lambda t^{-r} d \log \left(g^{-1}\right) \in \mathfrak{g}(\mathcal{O})\right\} / G(\mathcal{O}),
$$

where $d \log (g)$ is defined as in (3). Then $Y_{\widetilde{A}, 1}=Y_{A}$, and $Y_{\widetilde{A}, 0}$ is the affine Springer fiber of $\widetilde{A}$ defined by Kazhdan and Lusztig in [12] (see also [9]). Let us first show that

Lemma 3. For any $g G(\mathcal{O}) \in Y_{A}$,

$$
\left(\operatorname{Ad}_{g^{-1}}(\widetilde{A})-t^{-r} d \log \left(g^{-1}\right) \quad \bmod t\right) \in \mathfrak{g}(\mathcal{O}) / t \mathfrak{g}(\mathcal{O})=\mathfrak{g}
$$

is nilpotent.

Proof. Let $T$ be the maximal torus of $G$ whose Lie algebra is $\mathfrak{t}$. Let $X_{*}(T)$ be the coweight lattice of $T$ and $X_{*}(T)_{+}$be semi-group of dominant coweights. Each $\check{\lambda} \in$ $X_{*}(T)$ defines a point $t^{\check{\lambda}} \in T(F) \subset G(F)$. We have the Birkhoff decomposition

$$
G(F)=\bigsqcup_{\check{\lambda} \in X_{*}(T)_{+}} G(\mathcal{O}) t^{\check{\lambda}} G(\mathcal{O}) .
$$

Let $g \in G(F)$ be as in the lemma. We can write it as $g=g_{1} t^{\check{\lambda}} g_{2}$ for $g_{1}, g_{2} \in G(\mathcal{O})$ and a dominant coweight $\check{\lambda}$. Then we have

$$
\mathrm{Ga}_{g_{2}^{-1}}(B)=\mathrm{Ga}_{t^{\check{\lambda}}} \mathrm{Ga}_{g_{1}}(A) \text {. }
$$

It is clear that

$$
\begin{aligned}
& C=\mathrm{Ga}_{g_{1}}(A)=C_{r} t^{r}+C_{r+1} t^{r+1}+\cdots, \\
& D=\mathrm{Ga}_{g_{2}^{-1}}(B)=D_{r} t^{r}+D_{r+1} t^{r+1}+\cdots,
\end{aligned}
$$

with $C_{r}$ nilpotent. We need to show that $D_{r}$ is nilpotent.

Let $\mathfrak{g}=\sum_{i} \mathfrak{g}_{i}$ be the weight decomposition of $\mathfrak{g}$ with respect to $\check{\lambda}$. Then $\mathfrak{g}_{\geq 0}:=$ $\sum_{i \geq 0} \mathfrak{g}_{i}$ is a parabolic subalgebra of $\mathfrak{g}$, and $\mathfrak{g}_{>0}:=\sum_{i>0} \mathfrak{g}_{i}$ is its nil-radical and $\mathfrak{g}_{0}$ is a Levi subalgebra. Similarly, one has $\mathfrak{g}_{\leq 0}$ and $\mathfrak{g}_{<0}$. We observe that $X=X_{0}+X_{>0} \in$ $\mathfrak{g}_{0}+\mathfrak{g}_{>0}$ (resp. $X=X_{0}+X_{<0} \in \mathfrak{g}_{0}+\mathfrak{g}_{<0}$ ) is nilpotent in $\mathfrak{g}$ if and only if $X_{0}$ is nilpotent in $\mathfrak{g}_{0}$.

Now since $D=\operatorname{Ga}_{t^{\check{\lambda}}}(C)$, we know that $C_{r} \in \mathfrak{g}_{\geq 0}$ and $D_{r} \in \mathfrak{g}_{\leq 0}$. Furthermore, if we decompose $C_{r}=C^{\prime}+C^{\prime \prime}$ with $C^{\prime} \in \mathfrak{g}_{0}, C^{\prime \prime} \in \mathfrak{g}_{>0}$ and $D=D^{\prime}+D^{\prime \prime}$ with $D^{\prime} \in \mathfrak{g}_{0}, D^{\prime \prime} \in \mathfrak{g}_{<0}$, then $C^{\prime}=D^{\prime}$. Since $C_{r}$ is nilpotent, $C^{\prime}=D^{\prime}$ is nilpotent. Therefore $D_{r}$ is nilpotent.

By Lemma 2, Theorem 1 holds if there is a point $g G(\mathcal{O}) \in Y_{A}$ such that the above element is regular nilpotent. Such a point $g G(\mathcal{O})$ is called a regular point of $Y_{A}$ (cf. [8]). Therefore, the main theorem follows from 
Theorem 4. If $A_{r}$ is nilpotent (equivalently, $\widetilde{A} \bmod t$ is nilpotent) and $r \leq-2$, then $Y_{A}$ has a regular point.

Let us interpret this theorem more geometrically. Let $I$ be the Iwahori subgroup of $G(F)$, i.e. the pre-image of $B \subset G$ under the evaluation map $G(\mathcal{O}) \rightarrow G$, and $\mathcal{F} \ell=G(F) / I$ be the affine flag variety. Without loss of generality, we can assume that $\widetilde{A} \bmod t \in \mathfrak{n}$, where $\mathfrak{n}$ is the nil-radical of $\mathfrak{b}$. Let

$$
X_{A}=\left\{g \in G(F) \mid \operatorname{Ad}_{g^{-1}}(\widetilde{A})-t^{-r} d \log \left(g^{-1}\right) \in \operatorname{Lie} I\right\} / I \in \mathcal{F} \ell .
$$

There is a natural projection $\pi: X_{A} \rightarrow Y_{A}$. The following lemma is clear.

Lemma 5. A point $p=g G(\mathcal{O}) \in Y_{A}$ is a regular point if and only if $\pi^{-1}(p)$ consists of a single point.

Observe that to prove Theorem 4, it is enough to prove that $Y_{A} \cap \mathrm{Gr}^{0}$ has a regular point, where $\mathrm{Gr}^{0}$ is the neutral component of Gr. Let $\tilde{G}$ be the simply-connected cover of the derived group of $G$, and write $A=A_{0}+A_{1}$, where $A_{0} \in \operatorname{Lie}\left(Z(G)^{0}\right)(F)\left(Z(G)^{0}\right.$ being the neutral component of the center $Z(G)$ of $G)$ and $A_{1} \in \operatorname{Lie}(\tilde{G})(F)$. Then $Y_{A} \cap$ $\mathrm{Gr}^{0}$ is (topologically) isomorphic to $Y_{A_{1}}$, and $X_{A} \cap \mathcal{F} \ell^{0}$ is (topologically) isomorphic to $X_{A_{1}}$. In addition, the projection $\mathcal{F} \ell^{0} \rightarrow \mathrm{Gr}^{0}$ is (topologically) isomorphic to the map $\mathcal{F} \ell_{\tilde{G}} \rightarrow \mathrm{Gr}_{\tilde{G}}$, where $\mathcal{F} \ell_{\tilde{G}}\left(\right.$ resp. $\mathrm{Gr}_{\tilde{G}}$ ) denotes the affine flag variety (resp. affine Grassmannian) of $\tilde{G}$. Therefore, the map $X_{A} \cap \mathcal{F} \ell^{0} \rightarrow Y_{A} \cap \mathrm{Gr}^{0}$ is (topologically) isomorphic to $X_{A_{1}} \rightarrow Y_{A_{1}}$. Then according to Lemma 5 , it is sufficient to prove Theorem 4 for connected simply-connected semisimple algebraic groups. Hence, from now on, we will assume that $G$ is a connected simply-connected semisimple algebraic group.

An analogous statement for non-deformed affine Springer fibers has been proved in $[12] \S 4$. By imitating their proof, we find that it is sufficient to prove two propositions. The first one is the following:

Proposition 6. $Y_{A}$ is finite-dimensional.

Next, we formulate the second proposition. Recall that the affine Weyl group $W_{\text {aff }}$ of $G(F)$ acts on $H_{*}(\mathcal{F} \ell)$ (cf. [10] $\S 2.7$ ), where $H_{*}(\cdot)$ stands for the Borel-Moore homology.

Proposition 7. Assume that $A_{r}$ is nilpotent (possibly, equal to zero) and $r \leq-2$. Then the image of $H_{*}\left(X_{A}\right) \rightarrow H_{*}(\mathcal{F} \ell)$ is invariant under the action of the affine Weyl group $W_{\text {aff }}$.

For the sake of completeness, let us repeat the argument from [12] $\S 4$ that shows how the above two propositions imply Theorem 4.

Let $d=\operatorname{dim} X_{A}$ (it is finite by Proposition 6). Let $X$ be an irreducible component of $X_{A}$ of dimension $d$. Denote by $[X] \in H_{2 d}(\mathcal{F} \ell)$ the homology class represented by $X$. Then $[X] \neq 0$ by loc. cit. $\S 4$, Lemma 6 . Let $V_{A}$ be the image of $H_{2 d}\left(X_{A}\right) \rightarrow H_{2 d}(\mathcal{F} \ell)$. Then $V_{A}$ is generated by these $[X]$. By Proposition $7, V_{A}$ is a subrepresentation of the representation of $W_{\text {aff }}$ on $H_{2 d}(\mathcal{F} \ell)$. By loc. cit. $\S 4$, Lemma $8, V_{A}$ has a non-zero invariant vector under the action of the finite Weyl group $W_{\mathrm{f}} \subset W_{\text {aff }}$. For $i \in I_{\mathrm{f}}$, let $P_{i}$ be the parahoric subgroup of $G(F)$ with Lie algebra $\operatorname{Lie} P_{i}=\operatorname{Lie} I+\mathfrak{g}_{-\alpha_{i}}$. Let $\mathcal{F} \ell_{i}$ be the partial affine flag variety of parahoric subgroups of $G(F)$ which are conjugate 
to $P_{i}$, and $\pi_{i}: \mathcal{F} \ell \rightarrow \mathcal{F} \ell_{i}$ be the projection. Assume that $Y_{A}$ does not contain a regular point. Then for any $p=g I \in X_{A},\left(\operatorname{Ad}_{g^{-1}}(\widetilde{A})-t^{-r} d \log \left(g^{-1}\right) \bmod t\right)$ is an element of $\mathfrak{n}$ (by Lemma 3) which is not regular, and therefore is contained in the nil-radical of some parabolic subalgebra $\mathfrak{p}_{i}=\mathfrak{b}+\mathfrak{g}_{-\alpha_{i}}, i \in I_{\mathrm{f}}$. In this case, for any $g^{\prime} \in g P_{i},\left(\operatorname{Ad}_{g^{\prime-1}}(\widetilde{A})-t^{-r} d \log \left(g^{\prime-1}\right) \bmod t\right)$ is also contained in $\mathfrak{n}$ (in fact, in the nil-radical of $\left.\mathfrak{p}_{i}\right)$ and therefore $\pi_{i}^{-1}\left(\pi_{i}(p)\right) \subset X_{A}$. For each $d$-dimensional irreducible component $X \subset X_{A}$, let $X_{i}, i \in I_{\mathrm{f}}$ be the closed subset of points $p$ on $X$ such that $\pi_{i}^{-1}\left(\pi_{i}(p)\right) \subset X_{A}$. Then $X=\cup_{i \in I_{\mathrm{f}}} X_{i}$. Since $X$ is irreducible, $X=X_{i}$ for some $i$, i.e., there exists some $i \in I_{\mathrm{f}}$ such that $X=\pi_{i}^{-1}\left(\pi_{i}(X)\right)$. Let $T_{s_{i}}$ be the corresponding simple reflection in $W_{\mathrm{f}}$, which acts on $H_{2 d}(\mathcal{F} \ell)$. Then $\left(\mathrm{Id}+T_{s_{i}}\right)[X]=0$. Now let $T=\sum_{w \in W_{\mathrm{f}}} T_{w}$. Since for any $i \in I_{\mathrm{f}}, T=Q_{i}\left(\operatorname{Id}+T_{s_{i}}\right)$, we find that $T[X]=0$ for any $d$-dimensional irreducible component $X \subset X_{A}$. Therefore, $T V_{A}=0$, which contradicts the fact that $V_{A}$ has a non-zero invariant vector under the action of $W_{\mathrm{f}}$.

In the remaining part of this note we prove Propositions 6 and 7 about the deformed affine Springer fibers. We also discuss the action of the affine Weyl group on $H_{*}\left(X_{A}\right)$.

\section{Proof of Proposition 6}

We begin with the proof of Proposition 6. Recall the definition of $M_{A}$ from the beginning of last section. For any $g \in M_{A}$, we consider the following $\mathbb{C}$-vector space

$$
T_{g}=\left\{X \in \mathfrak{g}(F) \mid \partial_{t} X+[B, X] \in t^{r} \mathfrak{g}(\mathcal{O})\right\} / \mathfrak{g}(\mathcal{O})
$$

where $B=\mathrm{Ga}_{g^{-1}}(A)$. Observe that if $g^{\prime}=g g_{1}$ with $g_{1} \in G(\mathcal{O})$, then $B^{\prime}=$ $\mathrm{Ga}_{g^{\prime-1}}(A)=\mathrm{Ga}_{g_{1}^{-1}}(B)$, and there is a canonical isomorphism $\gamma_{g_{1}}: T_{g} \cong T_{g^{\prime}}$ given by $X \mapsto \operatorname{Ad}_{g_{1}^{-1}}(X)$. Therefore, $T_{g}$ is canonically attached to every $g G(\mathcal{O}) \in Y_{A}$. From the definition of $Y_{A}$, it is clear that $T_{g}$ is canonically isomorphic to the tangent space of $Y_{A}$ at $g G(\mathcal{O})$. We claim that the dimension of this $\mathbb{C}$-vector space is $\leq(-r) \operatorname{dim} \mathfrak{g}$. This proves that the dimension of $Y_{A}$ is $\leq(-r) \operatorname{dim} \mathfrak{g}$.

We regard $\mathfrak{g}(F)$ as a vector space over $F$, with a connection $\nabla_{t}=\partial_{t}+\operatorname{ad}(B)$. Then $T_{g}=\nabla^{-1}\left(t^{r} \mathfrak{g}(\mathcal{O})\right) / \mathfrak{g}(\mathcal{O})$. Now the claim is a direct consequence of the following lemma, whose proof was suggested to us by D. Arinkin.

Let $(V, \nabla)$ be a finite-dimensional vector space over $F$ with a connection. By an $\mathcal{O}$-lattice in $V$ we understand a finite generated $\mathcal{O}$-submodule $L$ of $V$ such that the natural map $L \otimes_{\mathcal{O}} F \rightarrow V$ is an isomorphism. By a lattice in $V$ we understand a $\mathbb{C}$-subspace in $V$ that is commensurable with an $\mathcal{O}$-lattice.

Lemma 8. For any lattice $L \subset V, \nabla^{-1}(L)$ is also a lattice of $V$, and the relative dimension of $\nabla^{-1}(L)$ to $L$ is

$$
\left[\nabla^{-1}(L): L\right]:=\operatorname{dim} \frac{\nabla^{-1}(L)}{\nabla^{-1}(L) \cap L}-\operatorname{dim} \frac{L}{\nabla^{-1}(L) \cap L} \leq 0 .
$$

Remark 5. This lemma is an easy consequence of Deligne's theory of "good lattices" for connections (cf. [3] pp.110-112), as we learned from D. Arinkin. However, to prove the existence of "good lattices", Deligne used the existence of the cyclic vector for $(V, \nabla)$ (cf. Remark 1). Therefore we prefer to avoid using these results in the proof of our theorem. 
Proof. We first recall that the connection $(V, \nabla)$ is said to be in the canonical form (with respect to some $F$-basis e of $V$ ), if it looks as follows:

$$
\partial_{t}+H_{1} t^{r_{1}}+H_{2} t^{r_{2}}+\cdots+H_{m} t^{r_{m}}+X t^{-1}
$$

where $r_{1}<r_{2}<\cdots<r_{m}<-1, H_{i}$ are diagonal matrices, $X$ is an upper triangular matrix, and $\left[H_{i}, X\right]=0$. It is proved in [1], $\S 6$ that, possibly after a finite field extension $E / F$, for every connection $(V, \nabla)$, there exists some $\left(E\right.$-)basis e of $V \otimes_{F} E$, such that this connection is in a canonical form with respect to this basis.

Now we begin to prove the lemma. Assume that $\operatorname{dim} V=n$. Let $E=F\left(t^{1 / d}\right)$ be a finite extension of $F$ and an $E$-basis $\mathbf{e}$ of $V \otimes_{F} E$ such that the connection $\nabla$ with respect to this basis is in the canonical form. Let $\Lambda=\mathcal{O}_{E} \mathbf{e}$, where $\mathcal{O}_{E}$ is the integral closure of $\mathcal{O}$ in $E . \Lambda$ is a lattice of $V \otimes_{F} E$. Since the connection is in the canonical form with respect to $\mathbf{e}$, we have:

$$
\nabla^{-1}\left(t^{k} \Lambda\right) \subset t^{k+1} \Lambda+\text { Sol, for any } k,
$$

where Sol $\subset V \otimes_{F} E$ is the kernel of $\nabla$ (that is, the solution space of $\nabla$ ). Note that $\operatorname{dim}_{\mathbb{C}}(\mathrm{Sol}) \leq n$.

Set $M=\Lambda \cap V$ inside $V \otimes_{F} E$. Then $M$ is a lattice in $V$. By (7),

$$
\left[\nabla^{-1}\left(t^{k} M\right): t^{k} M\right] \leq 0,
$$

because the codimension of $t^{k+1} M$ in $V \cap\left(t^{k+1} \Lambda+\mathrm{Sol}\right)$ is at most $n$.

Finally, we can prove the statement. Indeed, take any lattice $L$ and choose $k$ such that $L \supset t^{k} M$. Since

$$
\operatorname{dim}\left(L / t^{k} M\right) \geq \operatorname{dim}\left(\nabla^{-1} L / \nabla^{-1}\left(t^{k} M\right)\right),
$$

the statement follows.

Remark 6. Observe that the tangent spaces for the non-deformed affine Springer fiber are never finite-dimensional (even for regular semisimple elements in $\mathfrak{g}(F)$ ). This is because the non-deformed affine Springer fiber of a regular semisimple element is highly non-reduced and has infinitely many "nilpotent directions".

\section{Proof of Proposition 7}

Let $I_{\text {aff }}=I_{\mathrm{f}} \bigsqcup\left\{i_{0}\right\}$ be the set of vertices in the affine Dynkin diagram for $G(F)$, with $i_{0}$ corresponding to the affine vertex. Denote by $T_{s_{i}}$ to the simple reflection corresponding to the vertex $i \in I_{\text {aff }}$. It is enough to construct, for each $i$, an involution $\sigma_{i}: H_{*}\left(X_{A}\right) \rightarrow H_{*}\left(X_{A}\right)$ such that the natural map $j: H_{*}\left(X_{A}\right) \rightarrow H_{*}(\mathcal{F} \ell)$ satisfies $j\left(\sigma_{i}(x)\right)=T_{s_{i}} j(x)$.

Let $\operatorname{Aut}^{0}(D)$ be the group of automorphisms of $D=\operatorname{Spec} \mathcal{O}$. It is an extension of $\mathbb{G}_{m}$ by a pro-unipotent group $\operatorname{Aut}^{+}(D)$ (see, e.g., [6] $\S 6.2$ ). The Lie algebra $\operatorname{Der}^{0}(D)$ of $\operatorname{Aut}^{0}(D)$ is topologically spanned by $\left\{t^{n} \partial_{t} ; n \geq 1\right\}$ and the Lie algebra $\operatorname{Der}^{+}(D)$ of $\operatorname{Aut}^{+}(D)$ is topologically spanned by $\left\{t^{n} \partial_{t} ; n \geq 2\right\}$. $\operatorname{Aut}^{0}(D)$ acts on $G(F)$, and we can form the semi-direct product $G(F) \rtimes \operatorname{Aut}^{0}(D)$. We have

$$
\operatorname{Lie}\left(G(F) \rtimes \operatorname{Aut}^{0}(D)\right)=\mathfrak{g}(F) \oplus \operatorname{Der}^{0}(D) \quad \text { as vector spaces. }
$$

Obviously, the action of $\operatorname{Aut}^{0}(D)$ on $G(F)$ leaves $G(\mathcal{O})$ invariant. Therefore, it acts on Gr. We thus obtain an action of $G(F) \rtimes \operatorname{Aut}^{0}(D)$ on Gr. In a similar fashion, 
$G(F) \rtimes \operatorname{Aut}^{0}(D)$ acts on all the affine (partial) flag varieties of $G(F)$, as is seen from the following lemma.

A standard parahoric subgroup of $G(F)$ is a parahoric subgroup of $G(F)$ that contains $I$. For $i \in I_{\text {aff }}$, let $P_{i}$ be the standard minimal parahoric subgroup corresponding to $i$.

Lemma 9. The action of $\operatorname{Aut}^{0}(D)$ on $G(F)$ leaves $I, P_{i}, i \in I_{\text {aff }}$ invariant and therefore leaves all standard parahoric subgroups of $G(F)$ invariant.

Proof. Write $G(\mathcal{O})=G^{(1)}(\mathcal{O}) G$. The action of $\operatorname{Aut}^{0}(D)$ on $G(\mathcal{O})$ leaves $G^{(1)}(\mathcal{O})$ invariant and fixes $G$. Since, $I$ and $P_{i}, i \in I_{\mathrm{f}}$ are pre-images of subgroups of $G$ under the evaluation map $G(\mathcal{O}) \rightarrow G$, they are invariant under the action of $\operatorname{Aut}^{0}(D)$. It remains to show that $P_{i_{0}}$ is also invariant under the action of $\operatorname{Aut}^{0}(D)$, where $i_{0}$ is the affine vertex in the affine Dynkin diagram of $\mathfrak{g}$.

We have $\operatorname{Lie} P_{i_{0}}=\operatorname{Lie} I+t^{-1} \mathfrak{g}_{\theta}$, where $\mathfrak{g}_{\theta}$ the the root space corresponding to the highest root $\theta$. It is clear that $\left[\operatorname{Der}^{0}(D), t^{-1} \mathfrak{g}_{\theta}\right] \subset t^{-1} \mathbb{C}[[t]] \mathfrak{g}_{\theta} \subset \operatorname{Lie} P_{i_{0}}$. Therefore, the action of $\operatorname{Aut}^{0}(D)$ also leaves $P_{i_{0}}$ invariant. Since the standard parahoric subgroups are generated by some of the $P_{i}$ 's, the lemma follows.

Thus, elements in the Lie algebra $\operatorname{Lie}\left(G(F) \rtimes \operatorname{Aut}^{0}(D)\right)$ act on these affine (partial) flag varieties by vector fields. The zero sets of these vector fields are nothing but our deformed affine Springer fibers! The reason is the following. The group $G(F)$ acts on $\operatorname{Lie}\left(G(F) \rtimes \operatorname{Aut}^{0}(D)\right)$ via the adjoint representation. Let us denote this adjoint representation by $\widetilde{\mathrm{Ad}}$ to distinguish it from the adjoint representation of $G(F)$ on $\mathfrak{g}(F)$. Let

$$
\left(\widetilde{A}, t^{-r} \partial_{t}\right) \in \mathfrak{g}(F) \oplus \operatorname{Der}^{0}(D), \quad r \leq-1
$$

We have

Lemma 10. For $g \in G(F), \widetilde{\operatorname{Ad}_{g}}\left(\left(\widetilde{A}, t^{-r} \partial_{t}\right)\right)=\left(\operatorname{Ad}_{g}(\widetilde{A})-t^{-r}\left(\partial_{t} g\right) g^{-1}, t^{-r} \partial_{t}\right)$.

Proof. Let $B \in \mathfrak{g}(F)$. We have

$$
\begin{aligned}
{\left[\widetilde{\operatorname{Ad}}_{g}\left(\left(\widetilde{A}, t^{-r} \partial_{t}\right)\right), B\right] } & =\widetilde{\operatorname{Ad}}_{g}\left[\left(\widetilde{A}, t^{-r} \partial_{t}\right), \operatorname{Ad}_{g^{-1}}(B)\right] \\
& =\left[\operatorname{Ad}_{g}(\widetilde{A}), B\right]+\operatorname{Ad}_{g}\left[t^{-r} \partial_{t}, \operatorname{Ad}_{g^{-1}}(B)\right] \\
& =\left[\operatorname{Ad}_{g}(\widetilde{A}), B\right]+\operatorname{Ad}_{g}\left(\left[t^{-r} \partial_{t}\left(g^{-1}\right) g, \operatorname{Ad}_{g^{-1}}(B)\right]+\operatorname{Ad}_{g^{-1}}\left(t^{-r} \partial_{t} B\right)\right) \\
& =\left[\left(\operatorname{Ad}_{g}(\widetilde{A})-t^{-r}\left(\partial_{t} g\right) g^{-1}, t^{-r} \partial_{t}\right), B\right]
\end{aligned}
$$

Since $\mathfrak{g}$ is semisimple, this identity implies the desired formula.

Therefore, if $\widetilde{A}, r$ are as in the assumption of Theorem 4 , we obtain that the reduced algebraic variety $X_{A}^{\text {red }} \subset \mathcal{F} \ell$ underlying $X_{A}$ is the zero set of the vector field on $\mathcal{F} \ell$ obtained by the action of $\left(\widetilde{A}, t^{-r} \partial_{t}\right) \in \operatorname{Lie}\left(G(F) \rtimes \operatorname{Aut}^{0}(D)\right)$. Likewise, $Y_{A}^{\text {red }} \subset \mathrm{Gr}$ is the zero set of the corresponding vector field on $\mathrm{Gr}$. Let $\mathcal{F} \ell_{i}=G(F) / P_{i}$, and $\pi_{i}: \mathcal{F} \ell \rightarrow \mathcal{F} \ell_{i}$ be the projection. This is a $\mathbb{P}^{1}$-fibration. We will also define $X_{A, i}^{\text {red }}$ to be the zero set of the corresponding vector field on $\mathcal{F} \ell_{i}$. It is clear that the projection $\pi_{i}: \mathcal{F} \ell \rightarrow \mathcal{F} \ell_{i}$ restricts to $\pi_{i}: X_{A}^{\text {red }} \rightarrow X_{A, i}^{\text {red }}$. 
Now, under the assumptions of Theorem $4, r \leq-2$, and $\widetilde{A} \in \operatorname{Lie} I^{0}$, where $I^{0}=$ $[I, I]$ is the pro-unipotent radical of $I$. Therefore,

$$
\left(\widetilde{A}, t^{-r} \partial_{t}\right) \in \operatorname{Lie} I^{0} \oplus \operatorname{Der}^{+}(D)=\operatorname{Lie}\left(I^{0} \rtimes \operatorname{Aut}^{+}(D)\right) .
$$

Since $I^{0} \rtimes \operatorname{Aut}^{+}(D)$ is pro-unipotent, the vector field on $\mathcal{F} \ell$ (resp., on $\mathcal{F} \ell_{i}$ ) gives rise to an action of $\mathbb{G}_{a}$ on $\mathcal{F} \ell$ (resp., on $\mathcal{F} \ell_{i}$ ). Furthermore, the projection $\pi_{i}: \mathcal{F} \ell \rightarrow \mathcal{F} \ell_{i}$ is $\mathbb{G}_{a}$-equivariant. Now $X_{A, i}^{\text {red }}$ is just the fixed point set of this $\mathbb{G}_{a}$ action on $\mathcal{F} \ell_{i}$. Therefore, there is a fiberwise $\mathbb{G}_{a}$-action on $\pi_{i}^{-1}\left(X_{A, i}^{\text {red }}\right)$, which is a $\mathbb{P}^{1}$-fibration over $X_{A, i}^{\mathrm{red}}$, and $X_{A}^{\mathrm{red}}$ is just the fixed point set. Now the construction of [11] $\S 2$ gives us the desired involution $\sigma_{i}: H_{*}\left(X_{A}\right) \rightarrow H_{*}\left(X_{A}\right)$.

This completes the proof of Proposition 7 and hence of Theorem 4. Therefore Theorem 1 is now proved.

\section{The action of the affine Weyl group on $H_{*}\left(X_{A}\right)$}

We continue to assume that $G$ is a connected simply-connected semisimple complex algebraic group. Let $A$ be a regular semisimple nil-element in $\mathfrak{g}(F)$, i.e., $(\operatorname{ad} A)^{r} \rightarrow 0$ if $r \rightarrow \infty$, as defined in [12] $\$ 2$. According to loc. cit., this is equivalent to the property that $A$ is conjugate to an element of $\mathfrak{g}(\mathcal{O})$ whose reduction modulo $t$ is a nilpotent element of $\mathfrak{g}$. Let $\mathrm{Sp}_{A}$ be the non-deformed affine Springer fiber of $A$ in $\mathcal{F} \ell$. In [14] §5, Lusztig constructed an action of $W_{\text {aff }}$ on $H_{*}\left(\mathrm{Sp}_{A}\right)$. We show in this section that a similar construction can be applied to obtain an action of $W_{\text {aff }}$ on the homology of the deformed affine Springer fibers.

Let $\left(\widetilde{A}, t^{-r} \partial_{t}\right) \in \operatorname{Lie} I^{0} \oplus \operatorname{Der}^{+}(D)$. We will prove that the homology $H_{*}\left(X_{A}\right)$ itself admits an action of the affine Weyl group, where the simple reflection corresponding to $i$ will act on $H_{*}\left(X_{A}\right)$ by $\sigma_{i}$ constructed above. The only new result here is Proposition 12 , the counterpart of which for the usual affine Springer fiber is proved in [14] §5.4.

For every $J \varsubsetneqq I_{\text {aff }}$, let $P_{J}$ be the standard parahoric subgroup of $G(F)$, generated by $P_{i}, i \in J$. This is a pro-algebraic group. Let $P_{J}^{u}$ be its pro-unipotent radical, so that $G_{J}:=P_{J} / P_{J}^{u}$ is a reductive group. Let $\mathfrak{g}_{J}=\operatorname{Lie} G_{J}$. For example, if $J=I_{\mathrm{f}}$ is the set of vertices in the finite Dynkin diagram, then $P_{I_{\mathrm{f}}}=G(\mathcal{O}), P_{I_{\mathrm{f}}}^{u}=G^{(1)}(\mathcal{O})$ and $G_{I_{\mathrm{f}}}=G$. The construction is based on the following

Lemma 11. Let $J \varsubsetneqq I_{\text {aff }}$. Then for any $g \in P_{J}$ and $r \leq-2$ we have $t^{-r}\left(\partial_{t} g\right) g^{-1} \in$ $\operatorname{Lie} P_{J}^{u}$.

Proof. It is enough to show that in $\operatorname{Lie}\left(G(F) \rtimes \operatorname{Aut}^{0}(D)\right),\left[t^{-r} \partial_{t}, \operatorname{Lie} P_{J}\right] \subset \operatorname{Lie} P_{J}^{u}$. It is easy to see that

$$
t^{2} \mathfrak{g}(\mathcal{O}) \subset \operatorname{Lie} P_{J}^{u} \subset \operatorname{Lie} P_{J} \subset t^{-1} \mathfrak{g}(\mathcal{O})
$$

for any $J \subsetneq I_{\text {aff. }}$. First we assume that $i_{0} \notin J$. In this case, $\operatorname{Lie} P_{J} \subset \mathfrak{g}(\mathcal{O})$ and therefore $\left[t^{-r} \partial_{t}, \operatorname{Lie} P_{J}\right] \subset t^{2} \mathfrak{g}(\mathcal{O}) \subset \operatorname{Lie} P_{J}^{u}$. The lemma holds. Next, we assume that $J=\left\{i_{0}\right\} \cup J^{\prime}$, with $J^{\prime} \varsubsetneqq I_{\mathrm{f}}$. Then

$$
\operatorname{Lie} P_{J}=\operatorname{Lie} P_{J} \cap \mathfrak{g}(\mathcal{O})+\sum_{\beta \in \Delta_{J^{\prime}}^{+} \cup\{0\}} t^{-1} \mathfrak{g}_{\theta-\beta},
$$

where $\Delta_{J^{\prime}}^{+}$is the set of positive roots for $G_{J^{\prime}}$. Clearly, $\left[t^{-r} \partial_{t}, \operatorname{Lie} P_{J} \cap \mathfrak{g}(\mathcal{O})\right] \in$ Lie $P_{J}^{u}$. In addition, $\left[t^{-r} \partial_{t}, t^{-1} \mathfrak{g}_{\theta-\beta}\right]=t^{-r-2} \mathfrak{g}_{\theta-\beta}$, which belongs to Lie $P_{J}$. But since $t^{r+2} \mathfrak{g}_{\beta-\theta} \nsubseteq \subseteq$ Lie $P_{J}, t^{-r-2} \mathfrak{g}_{\theta-\beta}$ indeed belongs to Lie $P_{J}^{u}$. The lemma follows. 
This lemma may also be reformulated as follows: the induced action of $\mathrm{Aut}^{+}(D)$ on $G_{J}=P_{J} / P_{J}^{u}$ is trivial.

Let

$$
X_{A, J}=\left\{g \in G(F) \mid \operatorname{Ad}_{g^{-1}}(\widetilde{A})-t^{-r} d \log \left(g^{-1}\right) \in \operatorname{Lie} P_{J}\right\} / P_{J} \subset G(F) / P_{J}
$$

be the deformed Springer fiber in $G(F) / P_{J}$. By Lemma 11, this is well-defined. For example, if $J=I_{\mathrm{f}}$, then $X_{A, J}=Y_{A}$. The natural projection $\pi_{J}: \mathcal{F} \ell \rightarrow G(F) / P_{J}$ restricts to a map $\pi_{J}: X_{A} \rightarrow X_{A, J}$.

Let $\widetilde{\mathfrak{g}}_{J} \stackrel{p_{J}}{\longrightarrow} \mathfrak{g}_{J}$ be the Grothendieck alteration of $\mathfrak{g}_{J}$, which classifies pairs consisting of a Borel subalgebra of $\mathfrak{g}_{J}$ and an element contained in this subalgebra.

Proposition 12. There is a natural Cartesian diagram

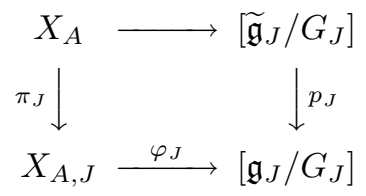

Proof. We first construct the morphisms $X_{A, J} \rightarrow \mathfrak{g}_{J} / G_{J}$. Let $\widetilde{X}_{A, J}$ be the preimage of $X_{A, J}$ under the projection $G(F) / P_{J}^{u} \rightarrow G(F) / P_{J}$. We have

$$
\widetilde{X}_{A, J}=\left\{g \in G(F) \mid \operatorname{Ad}_{g^{-1}}(\widetilde{A})-t^{-r} \partial_{t}\left(g^{-1}\right) g \in \operatorname{Lie} P_{J}\right\} / P_{J}^{u}
$$

By Lemma 11, the map

$$
g P_{J}^{u} \mapsto \operatorname{Ad}_{g^{-1}}(\widetilde{A})-t^{-r} \partial_{t}\left(g^{-1}\right) g \quad \bmod \operatorname{Lie} P_{J}^{u}
$$

is a well define $G_{J}$-equivariant map $\widetilde{X}_{A, J} \rightarrow \mathfrak{g}_{J}$. This gives the desired map $\varphi_{J}:$ $X_{A, J} \rightarrow \mathfrak{g}_{J} / G_{J}$.

Let $\widetilde{X}_{A}:=X_{A} \times_{X_{A, J}} \widetilde{X}_{A, J}$, so that $\widetilde{X}_{A}$ classifies the pairs $\left(g I, g^{\prime} P_{J}^{u}\right), g, g^{\prime} \in G(F)$ such that $g P_{J}=g^{\prime} P_{J}$ and

$$
\operatorname{Ad}_{g^{-1}}(\widetilde{A})-t^{-r} \partial_{t}\left(g^{-1}\right) g \in \operatorname{Lie} I, \quad \operatorname{Ad}_{g^{\prime-1}}(\widetilde{A})-t^{-r} \partial_{t}\left(g^{\prime-1}\right) g^{\prime} \in \operatorname{Lie} P_{J} .
$$

On the other hand, $\widetilde{\widetilde{X}}_{A}:=\widetilde{X}_{A, J} \times_{\mathfrak{g}_{J}} \widetilde{\mathfrak{g}}_{J}$ classifies pairs $\left(g I, g^{\prime} P_{J}^{u}\right), g \in P_{J}, g^{\prime} \in G(F)$ such that

$$
\operatorname{Ad}_{g^{\prime-1}}(\widetilde{A})-t^{-r} \partial_{t}\left(g^{\prime-1}\right) g^{\prime} \in \operatorname{Ad}_{g}(\operatorname{Lie} I) \subset \operatorname{Lie} P_{J}
$$

Let $\left(g I, g^{\prime} P_{J}^{u}\right) \in \widetilde{\widetilde{X}}_{A}$. We find that

$$
\operatorname{Ad}_{\left(g^{\prime} g\right)^{-1}}(\widetilde{A})-t^{-r} \partial_{t}\left(\left(g^{\prime} g\right)^{-1}\right)\left(g^{\prime} g\right)=\operatorname{Ad}_{g^{-1}}\left(\operatorname{Ad}_{g^{\prime-1}}(\widetilde{A})-t^{-r} \partial_{t}\left(g^{\prime-1}\right) g^{\prime}\right)-t^{-r} \partial_{t}\left(g^{-1}\right) g
$$

is in Lie $I$. This is because $\operatorname{Ad}_{g^{\prime-1}}(\widetilde{A})-t^{-r} \partial_{t}\left(g^{\prime-1}\right) g^{\prime} \in \operatorname{Ad}_{g}(\operatorname{Lie} I)$ and $t^{-r} \partial_{t}\left(g^{-1}\right) g \in$ Lie $P_{J}^{u} \subset \operatorname{Lie} I$ by Lemma 11 . Therefore, $\left(g^{\prime} g I, g^{\prime} P_{J}^{u}\right) \in \widetilde{X}_{A}$. Conversely, if $\left(g I, g^{\prime} P_{J}^{u}\right) \in$ $\widetilde{X}_{A}$, then $\left(g^{\prime-1} g I, g^{\prime} P_{J}^{u}\right) \in \widetilde{\widetilde{X}}_{A}$. Therefore, there is a $G_{J}$-equivariant isomorphism $\widetilde{X}_{A} \rightarrow \widetilde{\widetilde{X}}_{A}$ sending $\left(g I, g^{\prime} P_{J}^{u}\right) \rightarrow\left(g^{\prime-1} g I, g^{\prime} P_{J}^{u}\right)$.

Thus, we obtain a Cartesian diagram

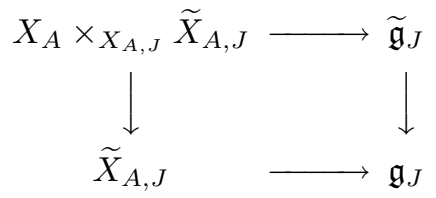


where all morphisms are $G_{J^{-}}$-equivariant. The proposition follows by taking the $G_{J^{-}}$ quotients.

Let $\underline{\mathbb{C}}$ be the constant sheaf on $X_{A}$. Then $\left(\pi_{J}\right)_{*} \mathbb{\mathbb { C }}=\varphi_{J}^{*}\left(p_{J}\right)_{*} \underline{\mathbb{C}}$. By the Springer theory for finite Weyl group, we obtain an action of $W_{J}$ (the finite Weyl group of $G_{J}$ ) on $\left(\pi_{J}\right)_{*} \mathbb{C}$. Therefore, we obtain a representation of $W_{J}$ on $H_{*}\left(X_{A}\right)$. Following the argument of [14] $\S 5.5$, we obtain that these representations for all $J \varsubsetneqq I_{\text {aff }}$ give rise to a representation of $W_{\text {aff }}$ on $H_{*}\left(X_{A}\right)$.

\section{Acknowledgments}

We thank D. Arinkin for suggesting a simpler proof of Proposition 6. E.F. thanks Fondation Sciences Mathématiques de Paris for its support and the group "Algebraic Analysis" at Université Paris VI for hospitality. X.Z. thanks Zhiwei Yun for useful discussions.

\section{References}

[1] D. Babbitt and V. Varadarajan, Formal reduction theory of meromorphic differential equations a group theoretic view, Pacific J. Math. 109 (1983), no. 1, 1-80.

[2] A. Beilinson and V. Drinfeld, Opers, Preprint, math.AG/0501398.

[3] P. Deligne, Equations Différentielles á Points Singuliers réguliers, Vol. 163 of Lect. Notes in Math., Springer (1970).

[4] E. Frenkel, Langlands Correspondence for Loop Groups, Vol. 103 of Cambridge Studies in Advanced Mathematics, Cambridge University Press, Cambridge (2007).

[5] - Ramifications of the geometric Langlands program, in Representation Theory and Complex Analysis, Vol. 1931 of Lect. Notes in Math., Springer Verlag (2008).

[6] E. Frenkel and D. Ben-Zvi, Vertex Algebras and Algebraic Curves, Vol. 88 of Mathematical Surveys and Monographs, AMS, 2nd edition (2004).

[7] E. Frenkel and D. Gaitsgory, Local geometric Langlands correspondence and affine Kac-Moody algebras, in Algebraic geometry and number theory, Vol. 253 of Progr. Math., 69-260, Birkháuser Boston, Boston, MA (2006).

[8] M. Goresky, R. Kottwitz, and R. MacPherson, Regular points in affine Springer fibers, Michigan Math. J. 53 (2005), no. 31, 97-107.

[9] M. Goresky, R. Kottwitz, and R. McPherson, Homology of affine Springer fibers in the unramified case, Duke Math. J. 121 (2004), no. 3, 509-561.

[10] V. Kac, Constructing groups associated to infinite-dimensional algebras, in Infinite Dimensional Groups with Applications, MSRI Publications, 167-216, Springer Verlag, Berlin (1985).

[11] D. Kazhdan and G. Lusztig, A Topological Approach to Springer's Representations, Adv. in Math. 38 (1980) 222-228.

[12] - Fixed point varieties on affine flag manifolds, Israel J. Math. 62 (1988), no. 2, 129-168.

[13] B. Kostant, Lie group representations on polynomial rings, Amer. J. Math. 85 (1963) 327-402.

[14] G. Lusztig, Affine Weyl groups and conjugacy classes in Weyl groups, Transform. Groups 1 (1996), no. 1-2, 83-97.

Department of Mathematics, University of California, Berkeley, CA 94720, USA

E-mail address: frenkel@math.berkeley.edu

Department of Mathematics, Harvard University, Cambridge, MA 02138, USA

E-mail address: xinwenz@math.harvard.edu 